\title{
Approximation by Certain Linear Positive Operators of Two Variables
}

\author{
Afşin Kürşat Gazanfer ${ }^{1}$ and İbrahim Büyükyazıcı ${ }^{2}$ \\ ${ }^{1}$ Department of Mathematics, Faculty of Science and Art, Bülent Ecevit University, 67100 Zonguldak, Turkey \\ ${ }^{2}$ Department of Mathematics, Faculty of Science, Ankara University, 06100 Tandogan, Turkey
}

Correspondence should be addressed to Afşin Kürşat Gazanfer; afsinkursat@beun.edu.tr

Received 21 September 2013; Revised 15 April 2014; Accepted 23 April 2014; Published 13 May 2014

Academic Editor: Adem Kılıçman

Copyright ( 2014 A. K. Gazanfer and İ. Büyükyazıc1. This is an open access article distributed under the Creative Commons Attribution License, which permits unrestricted use, distribution, and reproduction in any medium, provided the original work is properly cited.

\begin{abstract}
We introduce positive linear operators which are combined with the Chlodowsky and Szász type operators and study some approximation properties of these operators in the space of continuous functions of two variables on a compact set. The convergence rate of these operators are obtained by means of the modulus of continuity. And we also obtain weighted approximation properties for these positive linear operators in a weighted space of functions of two variables and find the convergence rate for these operators by using the weighted modulus of continuity.
\end{abstract}

\section{Introduction and Preliminaries}

Let $x \in \mathbb{R}_{0}^{+}=[0, \infty)$ and $f \in C\left(\mathbb{R}_{0}^{+}\right)$. The well-known Chlodowsky polynomial of degree $n$, denoted by $C_{n}(f ; x)$, is

$$
\begin{array}{r}
C_{n}(f ; x)=\sum_{k=0}^{n} C_{n}^{k}\left(\frac{x}{\alpha_{n}}\right)^{k}\left(1-\frac{x}{\alpha_{n}}\right)^{k} f\left(\frac{k}{n} \alpha_{n}\right), \\
0 \leq x \leq \alpha_{n},
\end{array}
$$

where $\left(\alpha_{n}\right)$ is a sequence of positive numbers with the properties

$$
\lim _{n \rightarrow \infty} \alpha_{n}=\infty, \quad \lim _{n \rightarrow \infty} \frac{\alpha_{n}}{n}=0 .
$$

Some generalization of these polynomials in the onedimensional case may be found in $[1,2]$. Recently, in $[3,4]$ some approximation theorems for different positive linear operators in the space of continuous functions for one variable case are done.

Let $x \in \mathbb{R}_{0}^{+}$and $f \in C\left(\mathbb{R}_{0}^{+}\right)$. The modified Szász-Mirakjan operators denoted by $S_{n}\left(f ; \beta_{n}, \gamma_{n}, x\right)=S(f ; x)$ are

$$
S_{n}(f ; x)=e^{-\beta_{n} x} \sum_{j=0}^{\infty} \frac{\left(\beta_{n} x\right)^{k}}{k !} f\left(\frac{k}{\gamma_{n}}\right),
$$

where $\left(\beta_{n}\right)$ and $\left(\gamma_{n}\right)$ are given increasing and unbounded sequences of positive numbers such that

$$
\lim _{n \rightarrow \infty} \frac{1}{\gamma_{n}}=0, \quad \frac{\beta_{n}}{\gamma_{n}}=1+O\left(\frac{1}{\gamma_{n}}\right) .
$$

In [5], Walczak introduced the modified Szász-Mirakjan operators in the polynomial weighted spaces of functions of one and two variables. He investigated approximation properties of modified Szász-Mirakjan operators in the weighted space of continuous functions of two variables for which $\rho f$ is uniformly continuous and bounded on $\mathbb{R}_{+}^{2}=\mathbb{R}_{0}^{+} \times \mathbb{R}_{0}^{+}$, where $\rho(x, y)$ is a polynomial weight function. In [6], İspir and Atakut studied the theorems on convergence of $S_{n}(f ; x)$, defined by (1) to $f(x)$, in the weighted spaces of continuous and obtain the convergence rate of the operators by using the weighted modulus of continuity on all positive semiaxis. They also study the modified Szász-Mirakjan operators in the polynomial weighted spaces of functions of two variables.

In this study, inspired by the operators (1) and (3), we consider certain linear positive operators of functions of two variables. To this end, let $f \in C\left(\square_{\alpha_{n}}\right)$, where 
$\square_{\alpha_{n}}=\left\{(x, y): 0 \leq x \leq \alpha_{n}, 0 \leq y<\infty\right\}$ and define the linear positive operators $L_{n, m}(f ; x, y)$ in the following way:

$$
\begin{aligned}
& L_{n, m}(f ; x, y) \\
& =\sum_{k=0}^{n} \sum_{j=0}^{\infty} P_{n, k}\left(\frac{x}{\alpha_{n}}\right) Q_{j}\left(\beta_{m} y\right) f\left(\frac{k}{n} \alpha_{n}, \frac{j}{\gamma_{m}}\right), \\
& (x, y) \in \square_{\alpha_{n}}, \quad n, m \in \mathbb{N}
\end{aligned}
$$

with $P_{n, k}(x)=C_{n}^{k} x(1-x)^{n-k}$ and $Q_{j}(x)=e^{-x}\left(x^{j} / j !\right)$.

If we take $f(x, y):=g(x)$ and $f(x, y):=h(y)$ in (5), then the operators $L_{n, m}$ reduce to the Chlodowsky and Szász type operators, respectively. Approximation of functions of one or two variables by some positive linear operators in weighted spaces may be found in $[1,2,5-8]$.

In this paper, firstly we study some approximation properties of the sequence of linear positive operators given by (5) in the space of continuous functions on compact set $\square_{A B}=[0, A] \times[0, B]$ and find the order of this approximation using full and partial modulus of continuity. We finally investigate the convergence of the sequence of linear positive operators $L_{n, m}$, defined on a weighted space of functions of two variables, and find the rate of this convergence by means of weighted modulus of continuity.

Now we give some basic definitions which we will use in our theorems.

Let $\rho(x, y)=1+x^{2}+y^{2}$ and let $B_{\rho}$ be the set of all functions $f$ defined on the real axis satisfying the condition $|f(x, y)| \leq M_{f} \rho(x, y)$, where $M_{f}$ is a constant depending only on $f . B_{\rho}$ is a normed space with the norm

$$
\|f\|_{\rho}=\sup _{(x, y) \in \mathbb{R}_{+}^{2}} \frac{|f(x, y)|}{\rho(x, y)} .
$$

Let $f \in C_{\rho}$ with $C_{\rho}$ being the subspace of all continuous functions belonging to $B_{\rho}$. Then the weighted modulus of continuity of $f$ defined by

$$
\Omega_{f}\left(\delta_{1}, \delta_{2}\right)=\sup _{\substack{\left|h_{1}\right| \leq \delta_{1},\left|h_{2}\right| \leq \delta_{2} \\(x, y) \in \mathbb{R}_{+}^{2}}} \frac{f\left(x+h_{1}, y+h_{2}\right)-f(x, y)}{\rho(x, y) \rho\left(h_{1}, h_{2}\right)} .
$$

Let $f \in C\left(\square_{A B}\right)$. The full modulus of continuity of $f$ is defined as follows:

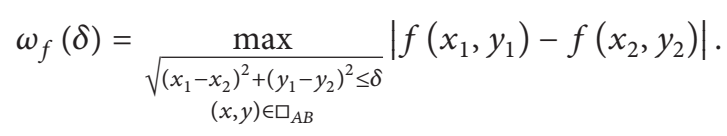

Partial modulus of continuity with respect to $x$ and $y$ is defined by

$$
\begin{aligned}
& \omega_{f}^{(1)}(\delta)=\max _{0 \leq y \leq B} \max _{\left|x_{1}-x_{2}\right| \leq \delta}\left|f\left(x_{1}, y\right)-f\left(x_{2}, y\right)\right|, \\
& \omega_{f}^{(2)}(\delta)=\max _{0 \leq x \leq A} \max _{\left|y_{1}-y_{2}\right| \leq \delta}\left|f\left(x, y_{1}\right)-f\left(x, y_{2}\right)\right|,
\end{aligned}
$$

respectively. It is known that the full modulus of continuity and the partial modulus of continuity satisfy the following properties:

$$
\begin{gathered}
\omega_{f}(\lambda \delta) \leq(1+\lambda) \omega_{f}(\delta), \quad \lim _{\delta \rightarrow 0} \omega_{f}(\delta)=0, \\
\omega_{f}(\delta) \leq \omega_{f}^{(1)}(\delta)+\omega_{f}^{(2)}(\delta) \leq 2 \omega_{f}(\delta) .
\end{gathered}
$$

\section{Approximation Properties on $\square_{A B}$}

In this section we give some classical approximation properties of the operators $L_{n, m}$ on the compact set $\square_{A B}$.

Let $e_{i, j}\left(t_{1}, t_{2}\right)=t_{1}^{i} t_{2}^{j}$. Then by simple calculations, one can obtain the following lemmas.

Lemma 1. Let $L_{n, m}$ be defined by (5). Then one has for all $m, n \in \mathbb{N}$,

$$
\begin{aligned}
& L_{n, m}\left(e_{0,0} ; x, y\right)=1 \text {, } \\
& L_{n, m}\left(e_{1,0} ; x, y\right)=x \text {, } \\
& L_{n, m}\left(e_{0,1} ; x, y\right)=\frac{\beta_{m}}{\gamma_{m}} y \text {, } \\
& L_{n, m}\left(e_{2,0} ; x, y\right)=\left(1-\frac{1}{n}\right) x^{2}+\frac{\alpha_{n}}{n} x \text {, } \\
& L_{n, m}\left(e_{0,2} ; x, y\right)=\frac{\beta_{m}^{2}}{\gamma_{m}^{2}} y^{2}+\frac{\beta_{m}}{\gamma_{m}^{2}} y, \\
& L_{n, m}\left(e_{3,0} ; x, y\right) \\
& =\left(\frac{2}{n^{2}}-\frac{3}{n}+1\right) x^{3}+\left(\frac{3 \alpha_{n}}{n}-\frac{3 \alpha_{n}}{n^{2}}\right) x^{2}+\frac{\alpha_{n}^{2}}{n^{2}} x, \\
& L_{n, m}\left(e_{0,3} ; x, y\right)=\frac{\beta_{m}^{3}}{\gamma_{m}^{3}} y^{3}+\frac{3 \beta_{m}^{2}}{\gamma_{m}^{3}} y^{2}+\frac{\beta_{m}}{\gamma_{m}^{3}} y \text {, } \\
& L_{n, m}\left(e_{4,0} ; x, y\right)=\left(1-\frac{6}{n}+\frac{11}{n^{2}}-\frac{6}{n^{3}}\right) x^{4} \\
& +\left(\frac{6 \alpha_{n}}{n}-\frac{18 \alpha_{n}}{n^{2}}+\frac{12 \alpha_{n}}{n^{3}}\right) x^{3} \\
& +\left(\frac{7 \alpha_{n}^{2}}{n^{2}}-\frac{7 \alpha_{n}^{2}}{n^{3}}\right) x^{2}+\frac{\alpha_{n}^{3}}{n^{3}} x \\
& L_{n, m}\left(e_{0,4} ; x, y\right)=\frac{\beta_{m}^{4}}{\gamma_{m}^{4}} y^{4}+\frac{6 \beta_{m}^{3}}{\gamma_{m}^{4}} y^{3}+\frac{7 \beta_{m}^{2}}{\gamma_{m}^{4}} y^{2}+\frac{\beta_{m}}{\gamma_{m}^{4}} y
\end{aligned}
$$

From Lemma 1, we obtain following lemmas.

Lemma 2. If the operator $L_{n, m}$ is defined by (5), then for all $(x, y) \in \mathbb{R}_{+}^{2}$ and $n, m \in \mathbb{N}$,

$$
\begin{gathered}
L_{n, m}\left(\left(e_{1,0}-x\right)^{2} ; x, y\right)=\frac{x\left(\alpha_{n}-x\right)}{n}, \\
L_{n, m}\left(\left(e_{0,1}-y\right)^{2} ; x, y\right)=\left(\frac{\beta_{m}}{\gamma_{m}}-1\right)^{2} y^{2}+\frac{\beta_{m}}{\gamma_{m}^{2}} y,
\end{gathered}
$$




$$
\begin{gathered}
L_{n, m}\left(\left(e_{1,0}-x\right)^{4} ; x, y\right) \\
=\left(\frac{3}{n^{2}}-\frac{6}{n^{3}}\right) x^{4}+\left(\frac{12 \alpha_{n}}{n^{3}}-\frac{6 \alpha_{n}}{n^{2}}\right) x^{3} \\
+\left(\frac{3 \alpha_{n}^{2}}{n^{2}}-\frac{7 \alpha_{n}^{2}}{n^{3}}\right) x^{2}+\frac{\alpha_{n}^{3}}{n^{3}} x, \\
L_{n, m}\left(\left(e_{0,1}-y\right)^{4} ; x, y\right) \\
=\left(\frac{\beta_{m}^{4}}{\gamma_{m}^{4}}-\frac{4 \beta_{m}^{3}}{\gamma_{m}^{3}}+\frac{6 \beta_{m}^{2}}{\gamma_{m}^{2}}-\frac{4 \beta_{m}}{\gamma_{m}}+1\right) y^{4} \\
+\left(\frac{6 \beta_{m}^{3}}{\gamma_{m}^{4}}-\frac{12 \beta_{m}^{2}}{\gamma_{m}^{3}}+\frac{6 \beta_{m}}{\gamma_{m}^{2}}\right) y^{3}+\left(\frac{7 \beta_{m}^{2}}{\gamma_{m}^{4}}-\frac{4 \beta_{m}}{\gamma_{m}^{3}}\right) y^{2} \\
+\frac{\beta_{m}}{\gamma_{m}^{4}} y .
\end{gathered}
$$

Lemma 3. If the operator $L_{n, m}$ is defined by (5), then for all $(x, y) \in \mathbb{R}_{+}^{2}$ and sufficiently large $n, m$,

$$
\begin{gathered}
L_{n, m}\left(\left(e_{1,0}-x\right)^{2} ; x, y\right)=O\left(\frac{\alpha_{n}}{n}\right)\left(x^{2}+x\right), \\
L_{n, m}\left(\left(e_{0,1}-y\right)^{2} ; x, y\right)=O\left(\frac{1}{\gamma_{m}}\right)\left(y^{2}+y\right), \\
L_{n, m}\left(\left(e_{1,0}-x\right)^{4} ; x, y\right)=O\left(\frac{\alpha_{n}}{n}\right)\left(x^{4}+x^{3}+x^{2}+x\right), \\
L_{n, m}\left(\left(e_{0,1}-y\right)^{4} ; x, y\right)=O\left(\frac{1}{\gamma_{m}}\right)\left(y^{4}+y^{3}+y^{2}+y\right) .
\end{gathered}
$$

The approximation theorem for functions of two variables is as follows.

Theorem 4 (see [8]). If $\left\{T_{n, m}\right\}$ is a sequence of linear positive operators satisfying the conditions

$$
\begin{gathered}
\lim _{n, m \rightarrow \infty}\left\|T_{n, m}\left(e_{0,0} ; x, y\right)-1\right\|_{C(X)}=0, \\
\lim _{n, m \rightarrow \infty}\left\|T_{n, m}\left(e_{1,0} ; x, y\right)-x\right\|_{C(X)}=0, \\
\lim _{n, m \rightarrow \infty}\left\|T\left(e_{0,1} ; x, y\right)-y\right\|_{C(X)}=0, \\
\lim _{n, m \rightarrow \infty}\left\|T_{n, m}\left(e_{1,0}^{2}+e_{0,1}^{2} ; x, y\right)-\left(x^{2}+y^{2}\right)\right\|_{C(X)}=0,
\end{gathered}
$$

then for any function $f \in C(X)$, which is bounded in $\mathbb{R}^{2}$,

$$
\lim _{n, m \rightarrow \infty}\left\|T_{n, m}(f ; x, y)-f(x, y)\right\|_{C(X)}=0,
$$

where $X$ is a compact set.

In the following theorem we show that the linear positive operator $L_{n, m}$ which we define by (5) converges to $f$ uniformly with the help of Theorem 4 given by Volkov in [8].
Theorem 5. Let $f \in C\left(\mathbb{R}_{+}^{2}\right)$, then the operators $L_{n, m}$ defined by (5) converge uniformly to $f$ on $\square_{A B} \subset \mathbb{R}_{+}^{2}$ as $n, m \rightarrow \infty$.

Proof. From (13)-(17) and conditions (2) and (4), we have

$$
\begin{gathered}
\lim _{n, m \rightarrow \infty}\left\|L_{n, m}\left(e_{0,0} ; x, y\right)-1\right\|_{C\left(\square_{A B}\right)}=0, \\
\lim _{n, m \rightarrow \infty}\left\|L_{n, m}\left(e_{1,0} ; x, y\right)-x\right\|_{C\left(\square_{A B}\right)}=0, \\
\lim _{n, m \rightarrow \infty}\left\|L_{n, m}\left(e_{0,1} ; x, y\right)-y\right\|_{C\left(\square_{A B}\right)}=0, \\
\lim _{n, m \rightarrow \infty}\left\|L_{n, m}\left(e_{1,0}^{2}+e_{0,1}^{2} ; x, y\right)-\left(x^{2}+y^{2}\right)\right\|_{C\left(\square_{A B}\right)}=0 .
\end{gathered}
$$

Applying Theorem 4, we obtain the desired result.

The following theorem gives the convergence rate of the sequence of linear positive operators $\left\{L_{n, m}\right\}$ to $f$ on $\square_{A B} C$ $\mathbb{R}_{+}^{2}$, by means of partial and full modulus of continuity.

Theorem 6. Let $f \in C\left(\square_{A B}\right)$; then the following inequalities hold:

$$
\begin{gathered}
\left\|L_{n, m}(f ; x, y)-f\right\|_{C\left(\square_{A B}\right)} \leq 2\left[\omega_{f}^{(1)}\left(\delta_{n}\right)+\omega_{f}^{(2)}\left(\delta_{m}\right)\right], \\
\left\|L_{n, m}(f ; x, y)-f\right\|_{C\left(\square_{A B}\right)} \leq 4 \omega_{f}^{(1)}\left(\delta_{n, m}\right),
\end{gathered}
$$

where $\delta_{n}=\sqrt{A\left(\alpha_{n} / n\right)}, \delta_{m}=\sqrt{B^{2}\left(\beta_{m}^{2} / \gamma_{m}^{2}\right)+B\left(\beta_{m} / \gamma_{m}^{2}\right)}, \delta_{n, m}=$ $\sqrt{A\left(\alpha_{n} / n\right)+B^{2}\left(\beta_{m}^{2} / \gamma_{m}^{2}\right)+B\left(\beta_{m} / \gamma_{m}^{2}\right)} ; \omega_{f}^{(1)}, \omega_{f}^{(2)}$, and $\omega_{f}$ are given by (9), (10), and (11), respectively.

Proof. From (5) and (13) and using the property of the partial modulus of continuity, we can write

$$
\begin{aligned}
& \left|L_{n, m}(f ; x, y)-f(x, y)\right| \\
& \quad \leq \sum_{k=0}^{n} \sum_{j=0}^{\infty} P_{n, k}\left(\frac{x}{\alpha_{n}}\right) Q_{j}\left(\beta_{m} x\right)\left|f\left(\frac{k}{n} \alpha_{n}, \frac{j}{\gamma_{m}}\right)-f\left(x, \frac{j}{\gamma_{m}}\right)\right| \\
& \quad+\sum_{k=0}^{n} \sum_{j=0}^{\infty} P_{n, k}\left(\frac{x}{\alpha_{n}}\right) Q_{j}\left(\beta_{m} x\right)\left|f\left(x, \frac{j}{\gamma_{m}}\right)-f(x, y)\right| \\
& \leq \omega_{f}^{(1)}\left(\delta_{n}\right)\left[1+\frac{1}{\delta_{n}} \sum_{k=0}^{n} P_{n, k}\left(\frac{x}{\alpha_{n}}\right)\left|\frac{k}{n} \alpha_{n}-x\right|\right] \\
& \quad+\omega_{f}^{(2)}\left(\delta_{m}\right)\left[1+\frac{1}{\delta_{m}} \sum_{j=0}^{\infty} Q_{j}\left(\beta_{m} x\right)\left|\frac{j}{\gamma_{m}}-y\right|\right] ;
\end{aligned}
$$


applying the Cauchy-Schwartz inequality, we have

$$
\begin{aligned}
& \left|L_{n, m}(f ; x, y)-f(x, y)\right| \\
& \leq \omega_{f}^{(1)}\left(\delta_{n}\right)\left[1+\frac{1}{\delta_{n}}\left(\sum_{k=0}^{n} P_{n, k}\left(\frac{x}{\alpha_{n}}\right)\left(\frac{k}{n} \alpha_{n}-x\right)^{2}\right)^{1 / 2}\right] \\
& \quad+\omega_{f}^{(2)}\left(\delta_{m}\right)\left[1+\frac{1}{\delta_{m}}\left(\sum_{j=0}^{\infty} Q_{j}\left(\beta_{m} x\right)\left(\frac{j}{\gamma_{m}}-y\right)^{2}\right)^{1 / 2}\right],
\end{aligned}
$$

and using equalities (16) and (17) and choosing $\delta_{n}=$ $\sqrt{A\left(\alpha_{n} / n\right)}, \delta_{m}=\sqrt{B^{2}\left(\beta_{m}^{2} / \gamma_{m}^{2}\right)+B\left(\beta_{m} / \gamma_{m}^{2}\right)}$, we obtain inequality (20). If we use inequality $\omega_{f}^{(1)}(\delta)+\omega_{f}^{(2)}(\delta) \leq 2 \omega_{f}(\delta)$ we can easily obtain inequality (21). Thus, the proof of theorem is completed.

Below in Example 7, we will try to see the agreement of our linear positive operator $L_{n, m}$ with $f(x, y)=2-(x-$ $1)^{2}+(y-1)^{2}$ using different values of $n$ and $m$, whereas in Example 8, we also compare $L_{n, m}$ with $S_{n, m}$ for another function $f(x, y)=x^{3} e^{-y} /\left(1+x^{4}\right)$.

Example 7. For $n, m=5, n, m=50$, and $\alpha_{n}=\sqrt{n}, \beta_{m}=$ $m, \gamma_{m}=m+\sqrt{m}$; the convergence of $L_{n, m}(f ; x, y)$ to $f(x, y)=$ $2-(x-1)^{2}+(y-1)^{2}$ is illustrated in Figure 1.

Example 8. For $n, m=30$ and $\alpha_{n}=\sqrt{n}, \beta_{m}=m$, $\gamma_{m}=m+\sqrt{m}$, the convergences of $L_{n, m}(f ; x, y)=$ $\sum_{k=0}^{n} \sum_{j=0}^{\infty} P_{n, k}\left(x / \alpha_{n}\right) Q_{j}\left(\beta_{m} x\right) f\left((k / n) \alpha_{n}, j / \gamma_{m}\right)$ and $S_{n, m}$ $(f ; x, y)=\sum_{k=0}^{\infty} \sum_{j=0}^{\infty} Q_{j}\left(\beta_{n} x\right) Q_{j}\left(\beta_{m} y\right) f\left(k / \gamma_{n}, j / \gamma_{m}\right)$ to $f(x, y)=x^{3} e^{-y} /\left(1+x^{4}\right)$ will be illustrated in Figure 2 .

\section{Weighted Approximation Properties of $L_{n, m}$}

In this section, we investigate the convergence properties of the $L_{n, m}$ operators given by (5) in the weighted spaces of continuous functions on positive semiaxis by using weighted Korovkin type theorem. The Korovkin type theorem in weighted spaces for linear positive operators $T_{n, m}$, acting from $C_{\rho}$ to $B_{\rho}$, has been proved by Gadjiev [9], Gadjiev and Hacısalihoğlu [10].

Theorem 9 (see [10]). There exists a sequence of positive linear operators $T_{n, m}$, acting from $C_{\rho}\left(\mathbb{R}_{+}^{2}\right)$ to $B_{\rho}\left(\mathbb{R}_{+}^{2}\right)$, satisfying the conditions

$$
\begin{gathered}
\lim _{n, m \rightarrow \infty}\left\|T_{n, m}(1 ; x, y)-1\right\|_{\rho}=0, \\
\lim _{n, m \rightarrow \infty}\left\|T_{n, m}\left(t_{1} ; x, y\right)-x\right\|_{\rho}=0, \\
\lim _{n, m \rightarrow \infty}\left\|T_{n, m}\left(t_{2} ; x, y\right)-y\right\|_{\rho}=0, \\
\lim _{n, m \rightarrow \infty}\left\|T_{n, m}\left(t_{1}^{2}+t_{2}^{2} ; x, y\right)-\left(x^{2}+y^{2}\right)\right\|_{\rho}=0 .
\end{gathered}
$$

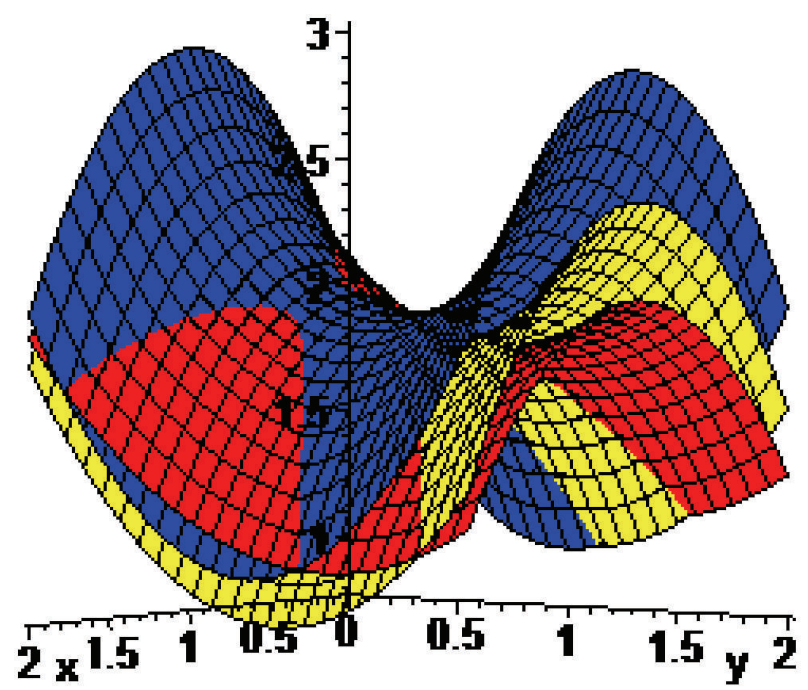

$f(x, y)$
$\square L_{50,50}(f ; x, y)$
$L_{5,5}(f ; x, y)$

Figure 1: The convergence of $L_{n, m}(f ; x, y)$ to $f(x, y)$ for $n, m=5$ and $n, m=50$.

Then there exists a function $f^{*} \in C_{\rho}\left(\mathbb{R}_{+}^{2}\right)$ for which

$$
\lim _{n, m \rightarrow \infty}\left\|T_{n, m} f^{*}-f^{*}\right\|_{\rho} \geq 1
$$

Theorem 10 (see [9]). Let $T_{n, m}$ be a sequence of positive linear operators acting from $C_{\rho}\left(\mathbb{R}_{+}^{2}\right)$ to $B_{\rho}\left(\mathbb{R}_{+}^{2}\right)$ and let $\rho_{1}(x, y) \geq 1$ be a continuous function for which

$$
\lim _{|v| \rightarrow \infty} \frac{\rho(v)}{\rho_{1}(v)}=0, \quad(\text { where } v=(x, y))
$$

The conditions (31)-(34) imply

$$
\lim _{n, m \rightarrow \infty}\left\|T_{n, m} f-f\right\|_{\rho_{1}}=0
$$

for all $f \in C_{\rho}\left(\mathbb{R}_{+}^{2}\right)$.

Now, we give the following results in $[9,10]$ which we use in the proofs of our main theorems.

Theorem 11. Let $L_{n, m}$ be the sequence of linear positive operators defined by (5). Then for all $f \in C_{\rho}\left(\mathbb{R}_{+}^{2}\right)$ one has

$$
\lim _{n, m \rightarrow \infty}\left\|L_{n, m} f-f\right\|_{\rho_{1}}=0
$$

where $\rho(x, y)=1+x^{2}+y^{2}$ and $\rho_{1}(x, y)$ is the continuous function, satisfying the condition (36). 


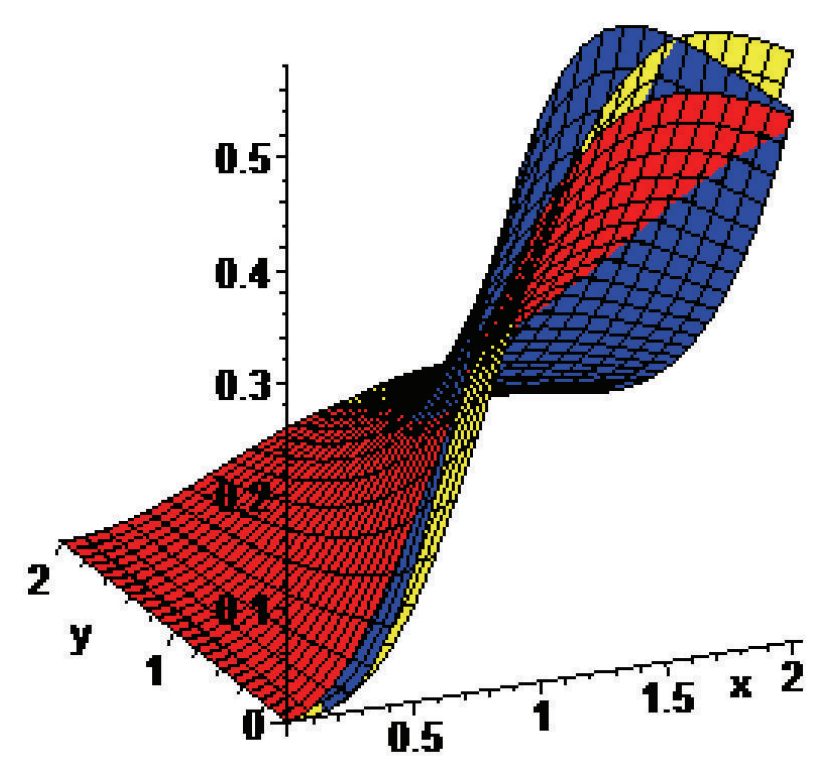

$f(x, y)$
$L_{30,30}(f ; x, y)$
$S_{30,30}(f ; x, y)$

FIgURE 2: The convergence of $L_{n, m}(f ; x, y)$ and $S_{n, m}(f, x, y)$ to $f(x, y)$ for $n, m=30$.

Proof. Firstly let us show that $L_{n, m}$ is acting from $C_{\rho}\left(\mathbb{R}_{+}^{2}\right)$ to $B_{\rho}\left(\mathbb{R}_{+}^{2}\right)$. Using (13), (16), and (17), we write

$$
\begin{aligned}
& \left\|L_{n, m}(\rho ; x, y)\right\|_{\rho} \\
& \leq 1+\sup _{(x, y) \in \mathbb{R}_{+}^{2}}\left[\left(1-\frac{1}{n}\right) \frac{x^{2}}{1+x^{2}+y^{2}}+\frac{\alpha_{n}}{n} \frac{x}{1+x^{2}+y^{2}}\right] \\
& \quad+\sup _{(x, y) \in \mathbb{R}_{+}^{2}}\left[\frac{\beta_{m}^{2}}{\gamma_{m}^{2}} \frac{y^{2}}{1+x^{2}+y^{2}}+\frac{\beta_{m}}{\gamma_{m}^{2}} \frac{y}{1+x^{2}+y^{2}}\right] \\
& \leq 1+\frac{\alpha_{n}}{n}+\frac{\beta_{m}^{2}}{\gamma_{m}^{2}}+\frac{\beta_{m}}{\gamma_{m}^{2}} \leq 1+\sigma_{n, m}
\end{aligned}
$$

where $\sigma_{n, m}=\left(\alpha_{n} / n\right)+\left(\beta_{m}^{2} / \gamma_{m}^{2}\right)+\left(\beta_{m} / \gamma_{m}^{2}\right)$. Since $\sigma_{n, m} \rightarrow 0$ as $n, m \rightarrow \infty$, there is a positive constant $M$ such that $\sigma_{n, m}<M$ for all natural numbers $n$ and $m$. Hence we have

$$
\left\|L_{n, m}(\rho ; x, y)\right\|_{\rho} \leq 1+M
$$

From [10], we have $L_{n, m}: C_{\rho}\left(I R_{+}^{2}\right) \rightarrow B_{\rho}\left(I R_{+}^{2}\right)$. If we prove that the conditions (31)-(34) are satisfied then the proof is completed by Theorem 10. By using (13), (14), and (15), we get (31)-(33). Finally, using (16) and (17), we have

$$
\left\|L_{n, m}\left(t_{1}^{2}+t_{2}^{2} ; x, y\right)-\left(x^{2}+y^{2}\right)\right\|_{\rho} \leq \sigma_{n, m},
$$

where $\sigma_{n, m}=\left(\alpha_{n} / n\right)+\left(\beta_{m}^{2} / \gamma_{m}^{2}\right)+\left(\beta_{m} / \gamma_{m}^{2}\right)$, and $\sigma_{n, m} \rightarrow 0$ as $n, m \rightarrow \infty$. Thus we obtain the desired result.

Theorem 12. Let $f \in C_{\rho}^{k}$. For sufficiently large $n, m$, the inequality

$$
\sup _{(x, y) \in \mathbb{R}_{+}^{2}} \frac{\left|L_{n, m}(f ; x, y)-f(x, y)\right|}{\rho^{3}(x, y)} \leq K \Omega_{f}\left(\delta_{n}, \delta_{m}\right)
$$

holds, where $\delta_{n}=\alpha_{n} / n, \delta_{m}=1 / \gamma_{m}$, and $K$ is a constant independent of $\alpha_{n}, \beta_{m}$, and $\gamma_{m}$.

Proof. From [6, page 577], we can write

$$
\begin{aligned}
& \left|f\left(t_{1}, t_{2}\right)-f(x, y)\right| \\
& \leq 8\left(1+x^{2}+y^{2}\right) \Omega_{f}\left(\delta_{n}, \delta_{m}\right)\left(1+\frac{\left|t_{1}-x\right|}{\delta_{n}}\right) \\
& \quad \times\left(1+\frac{\left|t_{2}-y\right|}{\delta_{m}}\right)\left(1+\left(t_{1}-x\right)^{2}\right)\left(1+\left(t_{2}-y\right)^{2}\right) .
\end{aligned}
$$

Applying the $L_{n, m}$ operators on both sides of the above inequality, we have

$$
\begin{aligned}
& \mid L_{n, m}(f ; x, y)-f(x, y) \mid \\
& \leq 8\left(1+x^{2}+y^{2}\right) \Omega_{f}\left(\delta_{n}, \delta_{m}\right) \\
& \times \sum_{k=0}^{n} P_{n, k}\left(\frac{x}{\alpha_{n}}\right)\left(1+\frac{1}{\delta_{n}}\left|\frac{k}{n} \alpha_{n}-x\right|\right)\left(1+\left(\frac{k}{n} \alpha_{n}-x\right)^{2}\right) \\
& \times \sum_{j=0}^{\infty} Q_{j}\left(\beta_{m} y\right)\left(1+\frac{1}{\delta_{m}}\left|\frac{j}{\gamma_{m}}-y\right|\right)\left(1+\left(\frac{j}{\gamma_{m}}-y\right)^{2}\right) \\
& \leq 8\left(1+x^{2}+y^{2}\right) \Omega_{f}\left(\delta_{n}, \delta_{m}\right) \\
& \quad \times \sum_{k=0}^{n} P_{n, k}\left(\frac{x}{\alpha_{n}}\right)\left[1+\left(\frac{k}{n} \alpha_{n}-x\right)^{2}+\frac{1}{\delta_{n}}\left|\frac{k}{n} \alpha_{n}-x\right|\right. \\
&\left.\quad+\frac{1}{\delta_{n}}\left|\frac{k}{n} \alpha_{n}-x\right|\left(\frac{k}{\delta_{m}} \alpha_{n}-x\right)^{2}\right] \\
& \quad \times \sum_{j=0}^{\infty} Q_{j}\left(\beta_{m} y\right)\left[1+\left(\frac{j}{\gamma_{m}}-y\right)^{2}+\frac{1}{\delta_{m}}\left|\frac{j}{\gamma_{m}}-y\right|\right. \\
& \gamma_{m}
\end{aligned}
$$

then, by Cauchy-Schwarz inequality,

$$
\begin{aligned}
& \left|L_{n, m}(f ; x, y)-f(x, y)\right| \\
& \quad \leq 8\left(1+x^{2}+y^{2}\right) \Omega_{f}\left(\delta_{n}, \delta_{m}\right)
\end{aligned}
$$




$$
\begin{aligned}
\times & {\left[1+L_{n, m}\left(\left(e_{1,0}-x\right)^{2} ; x, y\right)\right.} \\
& +\frac{1}{\delta_{n}} \sqrt{L_{n, m}\left(\left(e_{1,0}-x\right)^{2} ; x, y\right)} \\
& \left.+\frac{1}{\delta_{n}} \sqrt{L_{n, m}\left(\left(e_{1,0}-x\right)^{2} ; x, y\right) L_{n, m}\left(\left(e_{1,0}-x\right)^{4} ; x, y\right)}\right] \\
\times & {\left[1+L_{n, m}\left(\left(e_{0,1}-y\right)^{2} ; x, y\right)\right.} \\
+ & \frac{1}{\delta_{m}} \sqrt{L_{n, m}\left(\left(e_{0,1}-y\right)^{2} ; x, y\right)} \\
& \left.+\frac{1}{\delta_{m}} \sqrt{L_{n, m}\left(\left(e_{0,1}-y\right)^{2} ; x, y\right) L_{n, m}\left(\left(e_{0,1}-y\right)^{4} ; x, y\right)}\right] ;
\end{aligned}
$$

from equalities (20)-(23), we obtain

$$
\begin{aligned}
& \left|L_{n, m}(f ; x, y)-f(x, y)\right| \\
& \leq 8\left(1+x^{2}+y^{2}\right) \Omega_{f}\left(\delta_{n}, \delta_{m}\right) \\
& \times\left[1+O\left(\frac{\alpha_{n}}{n}\right)\left(x^{2}+x\right)+\frac{1}{\delta_{n}} \sqrt{O\left(\frac{\alpha_{n}}{n}\right)\left(x^{2}+x\right)}\right. \\
& \left.\quad+\frac{1}{\delta_{n}} \sqrt{O\left(\frac{\alpha_{n}}{n}\right)\left(x^{2}+x\right) O\left(\frac{\alpha_{n}}{n}\right)\left(x^{4}+x^{3}+x^{2}+x\right)}\right] \\
& \times\left[1+O\left(\frac{1}{\gamma_{m}}\right)\left(y^{2}+y\right)+\frac{1}{\delta_{m}} \sqrt{O\left(\frac{1}{\gamma_{m}}\right)\left(y^{2}+y\right)}+\frac{1}{\delta_{m}}\right. \\
& \left.\quad \times \sqrt{O\left(\frac{1}{\gamma_{m}}\right)\left(y^{2}+y\right) O\left(\frac{1}{\gamma_{m}}\right)\left(y^{4}+y^{3}+y^{2}+y\right)}\right]
\end{aligned}
$$

Choosing $\delta_{n}=\alpha_{n} / n$ and $\delta_{m}=1 / \gamma_{m}$, for sufficiently large $n, m$, we get

$$
\begin{aligned}
& \left|L_{n, m}(f ; x, y)-f(x, y)\right| \\
& \quad \leq K\left(1+x^{2}+y^{2}\right)\left(1+x^{2}+y^{2}\right)^{2} \Omega_{f}\left(\frac{\alpha_{n}}{n}, \frac{1}{\gamma_{m}}\right)
\end{aligned}
$$

which is the desired result.

\section{Conflict of Interests}

The authors declare that there is no conflict of interests regarding the publication of this paper.

\section{References}

[1] A. D. Gadjiev, R. O. Efendiev, and E. Ibikli, "Generalized Bernstein-Chlodowsky polynomials," The Rocky Mountain Journal of Mathematics, vol. 28, no. 4, pp. 1267-1277, 1998.

[2] E. A. Gadjieva and E. Ibikli, "On generalization of BernsteinChlodowsky polynomials," Hacettepe Bulletin of Natural Sciences and Engineering, vol. 24, pp. 31-340, 1995.
[3] M. Mursaleen and A. Khan, "Generalized $q$-Bernstein-Schurer operators and some approximation theorems," Journal of Function Spaces and Applications, vol. 2013, Article ID 719834, 7 pages, 2013.

[4] M. Mursaleen, F. Khan, and A. Khan, "Statistical approximation for new positive linear operators of Lagrange type," Applied Mathematics and Computation, vol. 232, pp. 548-558, 2014.

[5] Z. Walczak, "On certain modified Szász-Mirakyan operators for functions of two variables," Demonstratio Mathematica, vol. 33, no. 1, pp. 91-100, 2000.

[6] N. İspir and Ç. Atakut, "Approximation by modified SzaszMirakjan operators on weighted spaces," Proceedings of the Indian Academy of Sciences: Mathematical Sciences, vol. 112, no. 4, pp. 571-578, 2002.

[7] F. Taşdelen, A. Olgun, and G. Başcanbaz-Tunca, "Approximation of functions of two variables by certain linear positive operators," Proceedings of the Indian Academy of Sciences: Mathematical Sciences, vol. 117, no. 3, pp. 387-399, 2007.

[8] V. I. Volkov, "On the convergence of sequences of linear positive operators in the space of continuous functions of two variable," Matematicheskii Sbornik, vol. 43, no. 85, p. 504, 1957 (Russian).

[9] A. D. Gadjiev, Linear Positive Operators in Weighted Space of Functions of Several Variables, N4, Izvestiya Academy of Sciences of Azerbaijan, 1980.

[10] A. D. Gadjiev and H. Hacisalihoğlu, Convergence of the Sequences of Linear Positive Operators, Ankara University Press, Ankara, Turkey, 1995 (Turkish). 


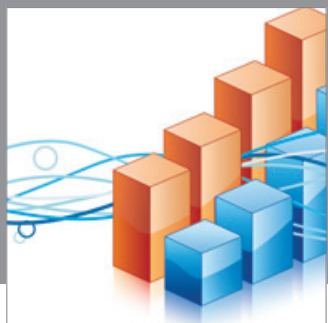

Advances in

Operations Research

mansans

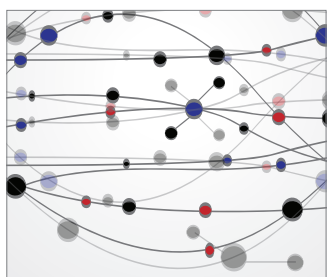

The Scientific World Journal
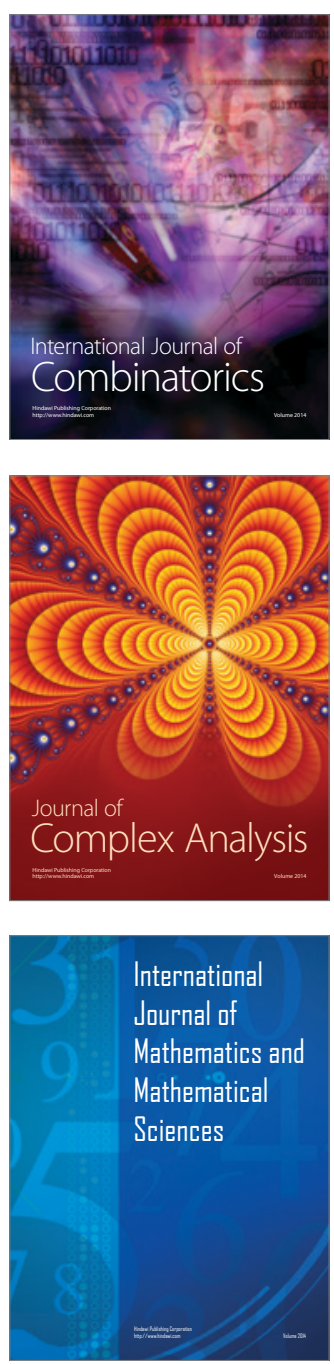
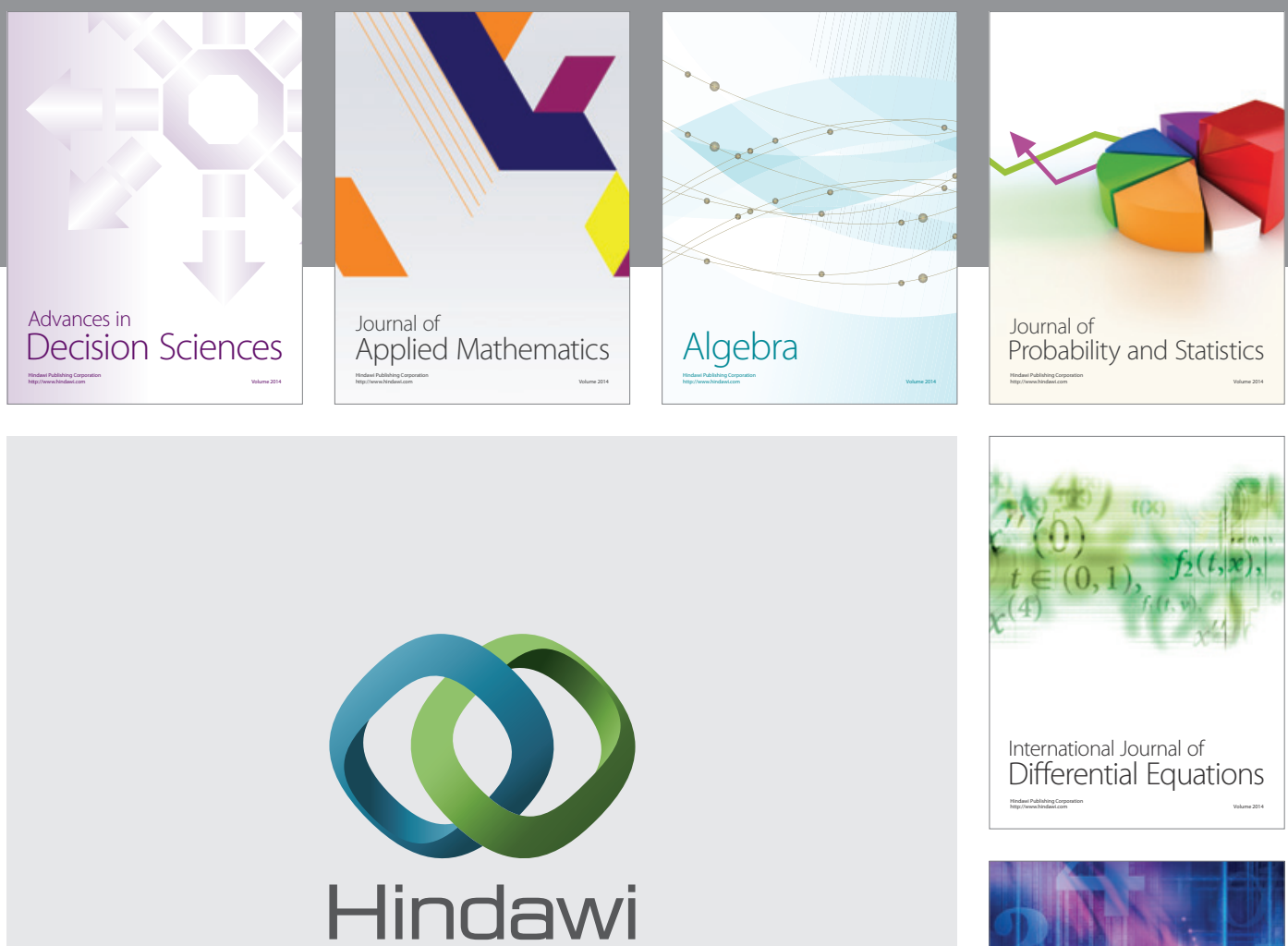

Submit your manuscripts at http://www.hindawi.com
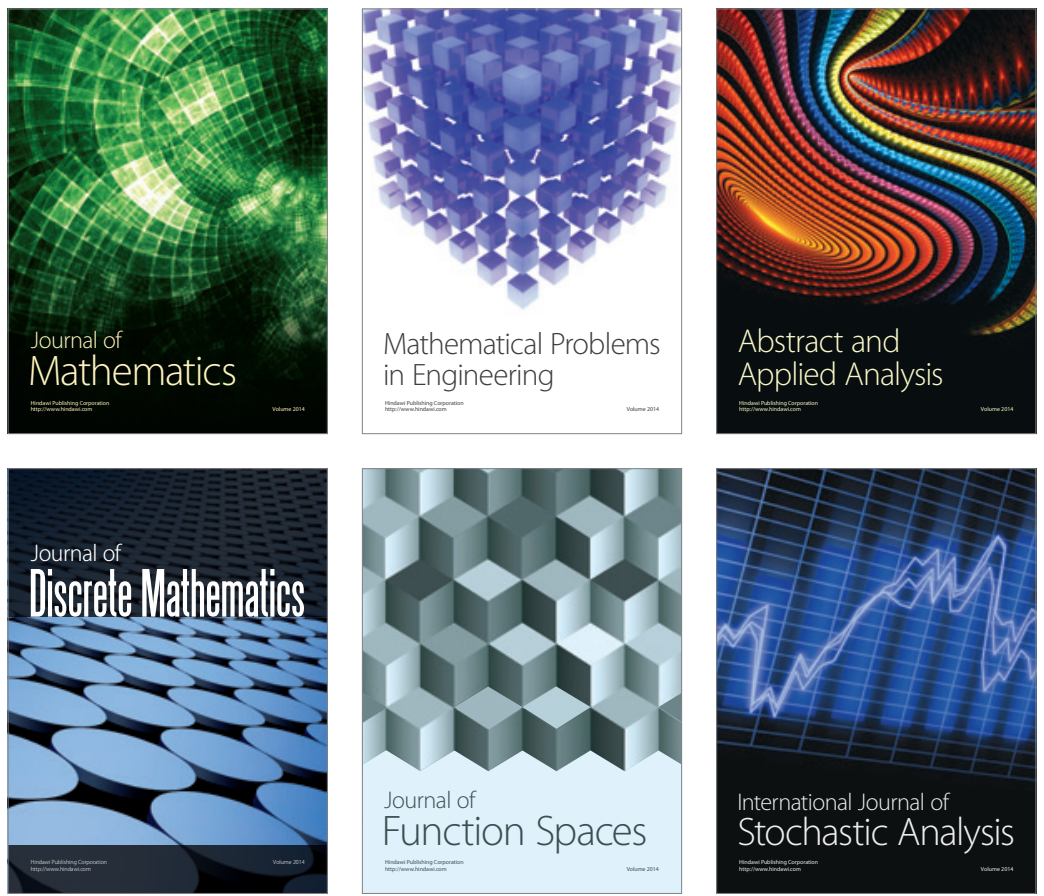

Journal of

Function Spaces

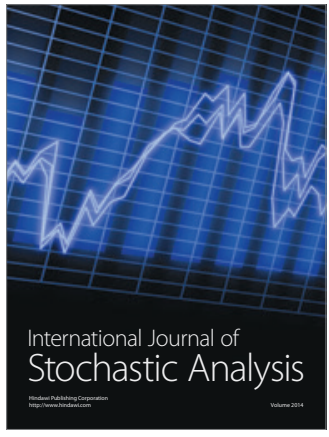

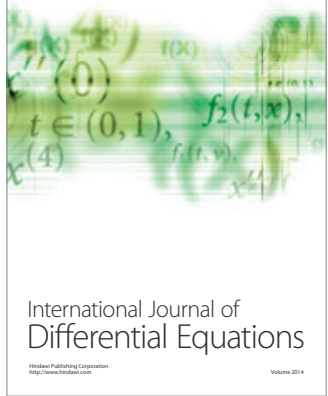
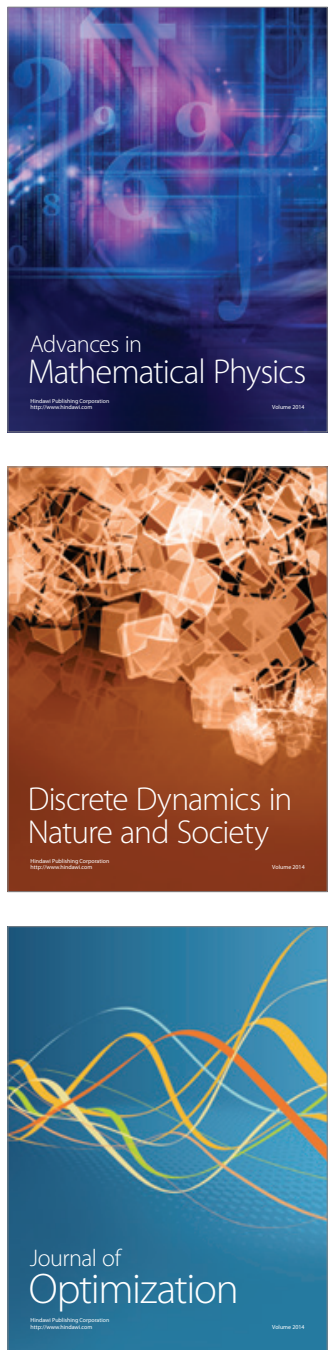